\title{
Diagnostic value of tachypnoea in pneumonia defined radiologically
}

\author{
Miguel Palafox, Héctor Guiscafré, Hortensia Reyes, Onofre Muñoz, Homero Martínez
}

\begin{abstract}
Objective-To evaluate whether sensitivity and specificity of tachypnoea for the diagnosis of pneumonia change with age, nutritional status, or duration of disease. Methods-Diagnostic testing of 110 children with acute respiratory infection, 51 of whom presented with tachypnoea. The gold standard was a chest roentgenogram. Thirty five children had a radiological image of pneumonia; 75 were diagnosed as not having pneumonia. Sensitivity, specificity, and percentage of correct classification of tachypnoea, by itself or in combination with other clinical signs for all children, by age groups, nutritional status, and disease duration were calculated.

Results-Tachypnoea as the sole clinical sign showed the highest sensitivity ( $74 \%)$ and a specificity of $67 \% ; 69 \%$ of cases were classified correctly. Sensitivity was reduced when other clinical signs were combined with tachypnoea, and there was no significant increase in correct classification, although specificity increased to $84 \%$. In children with a disease duration of less than three days, tachypnoea had a lower sensitivity and specificity (55\% and $64 \%$, respectively), and a lower percentage of correct classification (62\%). In children with low weight for age ( $<1 \mathrm{Z}$-score), tachypnoea had a sensitivity of $83 \%$, a specificity of $48 \%$, and $60 \%$ correct classification. Sensitivity and specificity did not vary with age groups.
\end{abstract}

Conclusions-Tachypnoea used as the only clinical sign is useful for identifying pneumonia in children, with no significant variations for age. In children with low weight for age, tachypnoea had higher sensitivity, but lower specificity. However, during the first three days of disease, the sensitivity, specificity, and percentage of correct classification were significantly lower.

(Arch Dis Child 2000;82:41-45)

Keywords: tachypnoea; pneumonia; sensitivity; specificity; diagnostic test

The World Health Organisation (WHO) has recommended tachypnoea as the most useful sign for identifying pneumonia in children. ${ }^{1}$ This sign may be easily detected, either by physicians or by lay people, including auxiliary health workers, parents, and other caregivers. Different field trials have shown that the identification of tachypnoea has been useful in diagnosing pneumonia, thus allowing early treatment for the sick child. ${ }^{2}{ }^{3}$ Clinical studies have shown that the lack of early referral is one of the most important risk factors for death in children with acute respiratory infections complicated with pneumonia, ${ }^{4}$ so early identification of the disease is a key aspect for survival.

Previous studies have shown that tachypnoea has high sensitivity and specificity to diagnose pneumonia. ${ }^{56}$ However, in the presence of severe disease, correlation with hypoxia is poor. Some authors have suggested that, in these cases, chest indrawing may be a better predictor. Some have also noted that the sensitivity and specificity of tachypnoea may be increased when combined with other clinical signs, like cough, flaring of the nose, or chest indrawing. Studies carried out in hospitals have also shown that the identification of alveolar rales has similar results to the identification of tachypnoea. $^{8}$

An aspect that has rarely been considered in the literature is whether tachypnoea has the same sensitivity and specificity to diagnose pneumonia in children $<6$ months of age, especially those under 2 months old, or in the presence of malnutrition, in which case the clinical signs may be less evident. In addition, the sensitivity and specificity of tachypnoea have not been evaluated in relation to different durations of disease.

The objective of our study was to evaluate whether the sensitivity and specificity of tachypnoea for the diagnosis of pneumonia changed in three different scenarios: early on in the disease; in young infants; and in the presence of malnutrition.

Material and methods

Our study was designed as a diagnostic test. It was carried out in a clinical unit of the General Hospital of the Ministry of Health, in the eastern state of Tlaxcala, Mexico. The unit was chosen because it is a referral centre for sick children in the state. Children between 3 days and 5 years of age were eligible for the study if they had needed medical care during the six month period of the study, had been clinically diagnosed with pneumonia, and had had the disease for less than two weeks. The clinical diagnosis was made by a paediatrician. Each child in the study had a matched control, who was the next child seen in the clinical unit with a diagnosis of acute respiratory infections, but without pneumonia. Acute respiratory infections was defined as the presence of a cough or rhinorrhea and signs of infectious syndrome of less than two weeks' duration. Children with chronic diseases, genetic abnormalities, neurological diseases, bronchial asthma, or septicaemia were not eligible. 
Table 1 Clinical characteristics of the 110 children studied

\begin{tabular}{|c|c|c|}
\hline & Number & $\%$ \\
\hline $\begin{array}{l}\text { Age (months) } \\
<2 \\
2-5 \\
6-11 \\
12-23 \\
24-59\end{array}$ & $\begin{array}{r}8 \\
23 \\
19 \\
31 \\
29\end{array}$ & $\begin{array}{r}7.3 \\
20.9 \\
17.3 \\
28.2 \\
26.3\end{array}$ \\
\hline $\begin{array}{l}\text { Duration of disease (days) } \\
<3 \\
3-5 \\
\geqslant 6\end{array}$ & $\begin{array}{l}37 \\
43 \\
30\end{array}$ & $\begin{array}{l}33.6 \\
39.1 \\
37.3\end{array}$ \\
\hline $\begin{array}{l}\text { Nutritional status } \\
\text { No weight for age deficit } \\
\text { With weight for age deficit } \\
\text { Mild ( }-1 \text { to }-2 \text { Z-score }) \\
\text { Moderate }(-2 \text { to }-3 \mathrm{Z} \text {-score }) \\
\text { Severe (> }-3 \mathrm{Z} \text {-score })\end{array}$ & $\begin{array}{r}77 \\
33 \\
25 \\
7 \\
1\end{array}$ & $\begin{array}{r}70 \\
30 \\
22.7 \\
6.4 \\
0.9\end{array}$ \\
\hline $\begin{array}{l}\text { Clinical data } \\
\text { Chest indrawing } \\
\text { Tachypnoea } \\
\text { Hyporexia } \\
\text { Fever } \\
\text { Alveolar rales } \\
\text { Wheezing } \\
\text { Drowsiness } \\
\text { Nasal flaring }\end{array}$ & $\begin{array}{r}56 \\
51 \\
42 \\
38 \\
32 \\
18 \\
11 \\
6\end{array}$ & $\begin{array}{r}50.9 \\
46.4 \\
38.2 \\
34.5 \\
29.1 \\
16.4 \\
10.6 \\
5.5\end{array}$ \\
\hline
\end{tabular}

The following symptoms were obtained from the clinical history: age, sex, duration of disease, and any symptoms referred by the mother. The clinical examination included documentation of the following: height, weight, body temperature, heart rate, respiratory rate, presence or absence of chest indrawing, alveolar rales, cyanosis, nose flaring, grunting, anorexia, wheezing, drowsiness, and laryngeal stridor. The respiratory rate was measured by observing for a full minute the chest movements, with the child lying down, without crying, and without fever. To measure the minute accurately, the physician used a one minute timer, distributed by UNICEF. The same paediatrician recorded all the clinical signs at two different times; the lowest value was the one recorded. Tachypnoea was defined according to criteria recommended by the WHO: in children less than 2 months old, respiratory rate $>60 /$ minute; in children between 2 and 12 months old, > 50/minute; and in children older than 12 months, $>40 /$ minute. $^{1}$

All children had a chest radiograph taken the same day as the consultation. The radiographs were evaluated by a single radiologist, who was blind to the clinical diagnosis. A diagnosis of pneumonia was made on the basis of the presence of micronodular or macronodular infiltrations or condensations in the lung. The $x$ ray results were taken as the gold standard. To assess intraobserver variability, the same radi- ologist evaluated 10 randomly selected radiographs twice, blind to the first diagnosis. The $\kappa$ statistic was calculated. ${ }^{9}$

\section{DATA ANALYSIS}

Sensitivity and specificity were calculated for each clinical sign and for all possible combinations, contrasted with the gold standard. Ninety five per cent confidence intervals were calculated, and significance was assessed by means of $\chi^{2}$ or Fisher's test, as appropriate. ${ }^{10} \mathrm{~A}$ discriminant analysis was carried out to evaluate the power of each clinical sign to identify pneumonia correctly. All analyses were run using SPSS software. ${ }^{11}$ Nutritional status was assessed according to weight for age deficit, taking standard deviations to classify mild, moderate, or severe weight for age losses, corresponding to $-1 \mathrm{Z}$-score, $-2 \mathrm{Z}$-score, or -3 Z-score, respectively, with National Centre for Health Statistics (NCHS) values as the reference population. ${ }^{12}{ }^{13}$

\section{Results}

Table 1 shows the characteristics of the 110 children included in our study. Only eight were younger than 2 months; over half were more than 1 year old. One third of the children had been sick for less than three days. Seventy seven had normal weight for age; eight showed moderate to severe weight for age losses. On the physical examination, 56 children had chest indrawing and 51 had tachypnoea. Fifty nine patients had a clinical diagnosis of pneumonia, but only 35 of them showed radiological signs of pneumonia. The $\kappa$ value calculated for intraobserver variability was 0.68 , which reflected a high degree of interobserver reliability.

Table 2 shows the clinical signs that, alone or combined, showed a sensitivity over $40 \%$ and a significant difference $(p<0.05)$. Only seven signs or combinations of signs were significant. Tachypnoea had the highest sensitivity $(74 \%)$, with an acceptable specificity (67\%), followed by chest indrawing. The association of both these signs improved specificity but not sensitivity. Alveolar rales had a higher specificity than these signs $(79 \%)$, but a very low sensitivity (46\%). Combinations of alveolar rales with tachypnoea or chest indrawing or a combination of these three signs improved specificity (80$84 \%$ ), but still had a very low sensitivity $(<46 \%)$. Tachypnoea was the clinical sign that showed the best percentage of correct classification of respiratory infection. Using discriminant analysis, we could show that a combination

Table 2 Sensitivity, specificity, and percentage of correct classification of clinical data compared with chest radiography

\begin{tabular}{|c|c|c|c|c|}
\hline Clinical sign & $\begin{array}{l}\% \text { Sensitivity } \\
(95 \% \mathrm{CI})\end{array}$ & $\begin{array}{l}\% \text { Specificity } \\
(95 \% \text { CI) }\end{array}$ & $\begin{array}{l}\% \text { Correct } \\
\text { classification }\end{array}$ & $p$ Value \\
\hline Tachypnoea & $74(60$ to 88$)$ & $67(56$ to 77$)$ & 69.1 & 0.00008 \\
\hline Chest indrawing & $71(56$ to 86$)$ & 59 (49 to 68$)$ & 62.7 & 0.004 \\
\hline Tachypnoea and chest indrawing & $68(52$ to 83$)$ & $69(58$ to 79$)$ & 62.7 & 0.0004 \\
\hline Tachypnoea and alveolar rales & $46(29$ to 62$)$ & $83(74$ to 91$)$ & 69.1 & 0.003 \\
\hline Alveolar rales & $46(29$ to 62$)$ & $79(70$ to 87$)$ & 68.2 & 0.01 \\
\hline Tachypnoea, chest indrawing, and alveolar rales & $43(26$ to 59$)$ & $84(75$ to 92$)$ & 62.7 & 0.001 \\
\hline Chest indrawing and alveolar rales & $42(25$ to 58$)$ & $80(71$ to 88$)$ & 68.2 & 0.02 \\
\hline All clinical data & $74(59$ to 87$)$ & $69(57$ to 76$)$ & 70.9 & 0.00006 \\
\hline Clinical judgment & $74(61$ to 88$)$ & $56(48$ to 69$)$ & 61.8 & 0.003 \\
\hline
\end{tabular}

$\%$ Correct classification was assessed by discriminant analysis. 
Table 3 Sensitivity, specificity, and percentage of correct classification of tachypnoea for children with pneumonia according to age, nutritional status, and time of onset of disease

\begin{tabular}{|c|c|c|c|c|c|}
\hline Clinical characteristic & $n$ & $\begin{array}{l}\% \text { Sensitivity } \\
(95 \% \mathrm{CI})\end{array}$ & $\begin{array}{l}\% \text { Specificity } \\
(95 \% \text { CI) }\end{array}$ & $\begin{array}{l}\% \text { Correct } \\
\text { classification }\end{array}$ & $p$ Value \\
\hline \multicolumn{6}{|l|}{ Age group (months) } \\
\hline$<6$ & 31 & $83(70$ to 96$)$ & 63 (46 to 80$)$ & 70.8 & \multirow{3}{*}{$>0.05$} \\
\hline 6 to 11 & 19 & $67(47$ to 87$)$ & $69(48$ to 90$)$ & 68.4 & \\
\hline 12 to 59 & 60 & 71 (59 to 82$)$ & $67(55$ to 79$)$ & 68.3 & \\
\hline \multicolumn{6}{|l|}{ Duration of disease (days) } \\
\hline$<3$ & 37 & 55 (40 to 72$)$ & $64(49$ to 80$)$ & 62.1 & \multirow{3}{*}{$<0.01$} \\
\hline 3 to 5 & 43 & 64 (49 to 78$)$ & $66(51$ to 80$)$ & 65.1 & \\
\hline$\geqslant 6$ & 30 & 93 (84 to 102$)$ & 73 (57 to 89$)$ & 83.3 & \\
\hline \multicolumn{6}{|l|}{ Nutritional status } \\
\hline No weight for age deficit & 77 & $69(50$ to 88$)$ & $74(62$ to 86$)$ & 72.7 & \multirow{2}{*}{0.44} \\
\hline With weight for age deficit & 33 & $83(62$ to 100$)$ & 48 (27 to 69$)$ & 60.6 & \\
\hline
\end{tabular}

\% Correct classification was assessed by discriminant analysis. CI, confidence interval.

of all the clinical signs only increased correct diagnosis in $1 \%$ of cases, compared with tachypnoea by itself. The physician's "clinical judgment" only gave $61.8 \%$ correct classification.

Table 3 shows sensitivity, specificity, and percentage of correct classification of respiratory infection using tachypnoea alone to identify pneumonia in children by age, weight for age deficits, and time of disease onset. There were no significant differences in relation to age. The percentage of correct classification was similar for the three age groups. Because

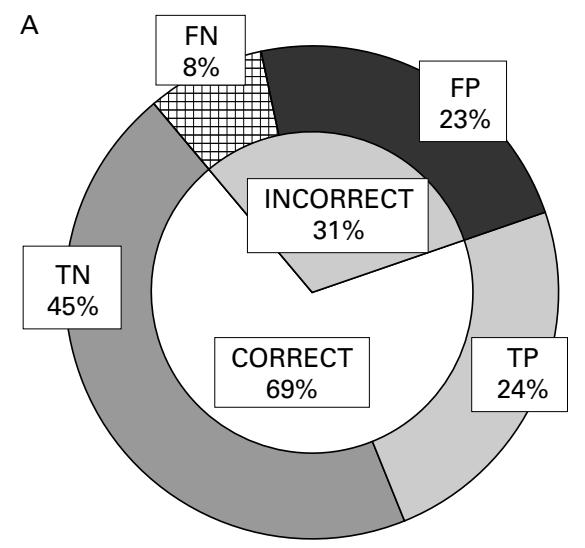

All 110 children

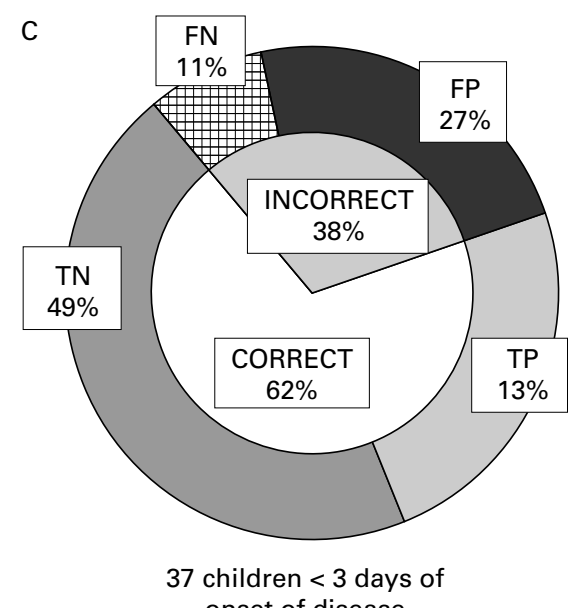

there were only eight children under 2 months of age, we considered it inappropriate to calculate significance of differences for this group.

On the other hand, we noted large differences in relation to the duration of the disease: The sensitivity, specificity, and per cent correct classification dropped to $55 \%, 64 \%$, and $62.1 \%$ when the duration of disease was less than three days, in contrast to $93 \%, 73 \%$, and $83.3 \%$, respectively, when the disease had lasted six or more days $(\mathrm{p}<0.01)$.

Figure 1 Graphic representation of the discriminant analysis for tachypnoea as the only sign to make a correct diagnosis of children with and without pneumonia (identified by chest roentgenogram) in 110 children with acute respiratory infection. TP, true positives; TN, true negatives; FP, false positives; FN, false negatives. 
In children with low weight for age, sensitivity increased to $83 \%$, but specificity dropped to $48 \%$. Thus, in children with low weight for age, there was a $60.6 \%$ correct classification of pneumonia, compared with $72.7 \%$ in children with no weight for age deficit $(p<0.05)$. In eight children with weight for age below the $-2 \mathrm{Z}$-score, sensitivity increased to $86 \%$ (data not shown), but it was not possible to evaluate specificity, because of the small number of children.

Figure 1 shows the discriminant analysis for tachypnoea as the diagnostic sign for pneumonia. When all 110 children were considered (fig 1A), pneumonia was identified correctly in $69 \%$ of cases. Forty five per cent were true negative and $24 \%$ were true positive. Thirty one per cent of cases identified erroneously as pneumonia by tachypnoea included $23 \%$ false positives and there were only $8 \%$ of children with pneumonia who did not have tachypnoea, or this sign was not identified (false negatives). In 31 children younger than 6 months (fig 1B) the percentage of correct classification was similar to the previous group, and false negatives only reached $6 \%$. In 37 children whose duration of illness was less than three days (fig 1C), only $62 \%$ were classified correctly, whereas false negatives increased to $11 \%$. Lastly, in 33 children with low weight for age (fig 1D), $61 \%$ were classified correctly, and false negatives were $6 \%$.

\section{Discussion}

In clinical practice, physicians tend to rely heavily on their clinical judgments to make a diagnosis. Specifically, to identify pneumonia, physicians look for the presence of alveolar rales audible through a stethoscope. In our study, we found that this sign had a high specificity $(79 \%)$, but that its sensitivity was very low (46\%). Therefore, its absence does not rule out the presence of pneumonia. In our study, we found that the "clinical judgment" of trained paediatricians identified pneumonia correctly in only $\sim 62 \%$ of all cases.

Previous clinical and field studies have shown that tachypnoea is the single most useful clinical sign for identifying pneumonia in children under 5 years of age. However, we have observed that, either in primary health care settings or in paediatric hospitals, the respiratory rate is commonly registered only in severely ill patients and it is often overlooked in children who apparently only have a mild episode of respiratory infection. In our study, we used criteria suggested by the WHO to classify tachypnoea, according to different age groups. ${ }^{1}$ We found that tachypnoea, by itself, had a sensitivity of $74 \%$ and a specificity of $67 \%$ to identify pneumonia, as has been reported by other authors, ${ }^{56}$ and was able to separate patients with pneumonia from those who did not have pneumonia in $69.1 \%$ of all cases. No other clinical sign, by itself or combined, performed better. In contrast to other authors, ${ }^{45}$ we did not find that combining tachypnoea with chest indrawing improved the sensitivity and specificity to diagnose pneumonia. The results from the discriminant analysis showed that not even all the clinical signs combined made a substantial improvement in sensitivity and specificity shown by tachypnoea in identifying pneumonia.

However, in $31 \%$ of cases, tachypnoea did not correctly identify children with or without pneumonia. In these cases, $23 \%$ were false positives and $8 \%$ were false negatives. Although it is possible that there is a true percentage of false positives, we should also consider that the gold standard that we used, a chest radiograph, may be subject to error. Although the same gold standard has been used by other authors in studies of respiratory infections, we should remember that the radiological picture of pneumonia appears 24-48 hours after the onset of disease. ${ }^{14}$ Therefore, it is likely that some cases that were considered false positives may have been in their earliest stages and therefore not detected by $x$ rays. ${ }^{15} 16$ Our study protocol did not consider a second chest radiograph, taken $48-72$ hours later, which would have allowed us to identify these cases. The false negative cases, even though only $8 \%$, should alert the clinician to consider that if the child does not have tachypnoea but presents other clinical signs suggestive of pneumonia, he or she may have the disease; thus, a chest roentgenogram should be taken, if possible, or the child should receive antibiotic treatment immediately.

Keeping these limitations of the gold standard in mind, we may now interpret the usefulness of tachypnoea in diagnosing pneumonia in relation to age, nutritional status, and disease duration. In children less than 6 months old we found that the sensitivity of tachypnoea was high, and because false negatives were only $6 \%$, we think there is strong support for giving antibiotic treatment to these children. However, there were too few cases of children under 2 months of age to give enough statistical power to our observations. In a previous study, Simoes et al found that tachypnoea was more difficult to assess in children under 2 months of age $^{17}$; more of these cases should be included in future studies.

In children with low weight for age $(<-1 \mathrm{Z}$ score) we also found a high sensitivity ( $83 \%)$, although specificity was low $(48 \%)$. Furthermore, in the eight children with weight for age deficits $<-2 \mathrm{Z}$-score, the sensitivity of tachypnoea was even higher $(86 \%)$. This finding contrasts with that of Falade et al, who found a sensitivity of $61 \%$ and a specificity of $79 \% .{ }^{18}$ However, these authors studied children with more severe weight for age deficits $(<70 \%$ of the mean NCHS standard, roughly equivalent to a $-3 \mathrm{Z}$-score). Based on our results, we think that tachypnoea is a useful sign to consider the diagnosis of pneumonia in malnourished children. Such a diagnosis is closely linked with the decision of whether or not to prescribe an antibiotic. In contrast, Falade et al suggest that antibiotics should be prescribed for all severely malnourished children who present with a cough or acute respiratory infection, regardless of their respiratory rate. Given the apparent discrepancy between these two recommendations, further studies should be carried out. At 
present, our recommendation seems to hold for children with a mild weight for age deficit, whereas the recommendation of Falade seems adequate for children with a severe weight for age deficit.

The most striking finding of our study was that, in children whose respiratory signs and symptoms had a duration of less than three days, sensitivity, specificity, and percentage of correct classification of pneumonia were significantly lower $(55 \%, 64 \%$, and $62 \%$, respectively), whereas false negatives were nearly doubled $(11 \%)$. The fact that radiological changes are still evolving during the early stages of the disease, making the gold standard less than perfect, would affect specificity (as a result of the increase of false negative cases), but this would not explain the concomitant reduction seen in sensitivity. Therefore, to rely exclusively on tachypnoea for identifying pneumonia would allow a large proportion of patients in the early stages of disease to go untreated, and the consequences of such misdiagnoses could be fatal. In these cases, we strongly recommend that the patient should be kept under a physician's supervision for 12-24 hours, or reassessed at this time as an outpatient. Likewise, complementary diagnostic tests should be carried out. We are not aware of previous studies that have investigated this issue.

In conclusion, we found that tachypnoea was the single most sensitive and specific sign for making a clinical diagnosis of pneumonia, and that it retained its sensitivity and specificity even in children under 6 months of age and with weight for age deficits. However, it had lower sensitivity and specificity in early stages of the disease if it was the sole diagnostic sign. In view of these data, we encourage physicians to use tachypnoea routinely to identify pneumonia in children, but to rely on it cautiously when the duration of disease is less than three days. In such cases, clinical signs should be monitored for at least 24-48 hours and, if in doubt, a chest roentgenogram should be taken, or antibiotic treatment prescribed without delay.

1 Programme for the control of acute respiratory infections. WHO/ARI/90.5, 1990:62-3.

2 Shann F, Hart K, Thomas D. Acute lower respiratory infections in children: possible criteria for selection of patients for antibiotic therapy and hospital admission. Bull World Health Organ 1984;62:749-53.

3 Harari M, Shann, F. Clinical signs of pneumonia in children. Lancet 1991;338:928-30.

4 Reyes H, Pérez-Cuevas R, Salmerón J, Tomé P, Guiscafré $\mathrm{H}$, Gutiérrez $\mathrm{G}$. Infant mortality due to acute respiratory infections: the influence of primary care processes. Health Policy and Planning 1997;12:214-23.

5 Singhi S, Dhawan A, Kataria S, Walia BNS. Validity of clinical signs for the identification of pneumonia in children. Ann Trop Paediatr 1994;14:53-8.

6 Gove S, Tulloch J, Cattani J, Schapira A. Usefulness of clinical case-definition in treatment of childhood malaria or pneumonia. Lancet 1993;341:304-5.

7 Berman S, Simoes E, Lanata C. Respiratory rate and pneumonia in infancy. Arch Dis Child 1991;66:81-4.

8 Mtango FDE, Neuvians D, Korte R. Magnitude, presentation, management and outcome of acute respiratory infections in children under the age of five in hospitals and rural tions in children under the age of five in hospitals and rural 102 .

9 Fleiss JL. The measurement of inter-rater agreement. In: Statistical methods for rates and proportions, 2nd ed. New York: John Wiley \& Sons, 1981:211-35.

10 Altman DG. In: Practical statistics for medical research. London: Chapman \& Hall, 1993:351-8.

11 SPSS professional statistic 6.1 edition. Chicago: SPSS Inc, 1993:1-45.

12 De Onís M, Habicht J-P. Anthropometric reference data for international use: recommendations from a World Health Organisation expert committee. Am f Clin Nutr 1996;64: 650-8.

13 Hamill P, Drizd T, Johnson CL, Reed RB. Health examination survey data from the National Center for Health Statistics (NCHS). Mon Vital Stat Rep 1976;25:1120.

14 Donowitz G, Mandell G. Acute pneumonia. In: Mandell G, Bennett J, Dolin R, eds. Principles and practices of infectious diseases, 4th ed. London: Churchill Livingstone, 1995:61937.

15 Leventhal JM. Clinical preditors of pneumonia as a guide to ordering chest roentgenograms. Clin Pediatr (Phila) 1982; 21:730-4.

16 Crain, EF, Bulas D, Bijur PE, Goldman HS. Is a chest radiograph necessary in the evaluation of every febrile infant less than 8 weeks of age? Pediatrics 1991;88:821-4.

17 Simoes EAF, Roark R, Berman S, Esler LL, Murphy J. Respiratory rate: measurement of variability over time and accuracy at different counting periods. Arch Dis Child 1991:66;1199-203.

18 Falade AB, Tschäppeler H, Greenwood BM, Mulholland EK. Use of simple clinical signs to predict pneumonia in young Gambian children: the influence of malnutrition. Bull World Health Organ 1995;73:299-304. 